\title{
Pathogenesis of pneumococcal infection
}

\author{
S.H. GILLESPIE and I. BALAKRISHNAN \\ Department of Medical Microbiology, Royal Free Campus, Royal Free and University College Medical School, \\ Rowland Hill Street, London NW3 2PF
}

\begin{abstract}
The pathogenesis of pneumococcal infection is a complex interplay between pneumococcal virulence determinants and the host immune response. Molecular studies have considerably advanced our knowledge and understanding of the precise structures and functions of the different determinants and their pathogenic roles. This review describes the mechanisms by which pneumococci attach, invade, evade lung defences and cause severe disease. Better understanding of the critical steps in this complex process will enable more effective clinical intervention to be developed to reduce the mortality exacted by this versatile pathogen.
\end{abstract}

\section{Introduction}

There has been an explosion in knowledge of Streptococcus pneumoniae pathogenicity. A constellation of possible virulence determinants has been described and several of these have been proposed as vaccine candidates. The role of the pneumococcal cell-wall polysaccharides in mediating adhesion and invasion and in generating inflammatory mediators is being recognised increasingly. The purpose of this review is to summarise the new information that has emerged on the pathogenesis of $S$. pneumoniae infection and the host immune response to it.

\section{Pathogenesis of severe infection}

S. pneumoniae has a collection of characteristics that permit it to colonise a variety of niches. Most pneumococci are found in the nasopharynx, a location where transformation can readily take place. This location also provides a convenient niche from which invasive disease can occur, and a complex interplay of factors determines whether the colonising organisms go on to establish infection of the lower respiratory tract. Colonising organisms may stimulate an immune response that eliminates them and the importance of this immune response is suggested by the observation that the duration of carriage is more protracted in children than in adults. Another factor that could influence the outcome of nasopharyngeal colonisation is the composition of microflora in this niche,

Received 14 Jan. 2000; accepted 2 May 2000.

Corresponding author: Professor S. H. Gillespie. facilitating or impeding colonisation and invasion by the relative degrees of symbiosis or competition.

Severe pneumococcal infection and its complications result partly from the direct actions of pneumococcal virulence determinants and partly from the host immune response to various pneumococcal components. Pneumococcal virulence determinants and the corresponding immune responses produce four key effects: adhesion, invasion, inflammation and shock.

\section{Adhesion}

Opacity. Changes in the colonial opacity of mucosal pathogens that alter their ability to colonise these surfaces have been described [1,2]. Colonial variants in $S$. pneumoniae were first recognised during the work that led to the understanding of transformation [3]. More recently, opacity variants have been described after culture on tryptose soy agar supplemented with catalase. Opaque colonies are larger, whiter, more uniform and domed. The bacterial cells with transparent colonies have surface structures that are less well defined and the cytoplasm appears to separate from the cell wall when they are examined by electron microscopy [4]. The organisms spontaneously alternate between the two phases at a rate of between $10^{-3}$ and $10^{-6}$. Transparent variants are able to colonise the nasopharynx in the infant rat model more easily than opaque variants and this is in keeping with their greater capacity for adherence to various cells, e.g., human buccal epithelial cells, human lung epithelial cells (type II pneumocytes) and vascular endothelial cells [5]. The adherence of transparent variants is enhanced by treatment of the target cells with interleukin-1 (IL-1) and tumour necrosis factor-alpha $(\mathrm{TNF}-\alpha)$. This is 
probably due to cytokine-induced expression of cellular platelet-activating factor (PAF) receptors, to which the transparent phenotype binds via a phosphorylcholine ligand (see below) [6].

The opacity locus has now been cloned and sequenced and its structure is illustrated in Fig. 1. The first open reading frame (ORF) contains the $g l p D$ gene, which encodes a 154 amino-acid protein with some similarity to the glycerol-3-phosphate dehydrogenase of Bacillus subtilis. The second ORF contains the glpF gene, which encodes a 233 amino-acid protein with $50 \%$ identity to the glycerol uptake facilitator of $B$. subtilis. Located $3^{\prime}$ to $g l p F$ are two repeated intergenic DNA elements, box A and box $\mathrm{C}-$ it is thought that these boxes associate to form a stem-loop structure. Although the strain can be either opaque or transparent without the box element, the frequency of phase variation increases 1000 -fold if it is present. A third open reading frame, ORF3, is located downstream from the box element - it is composed of small ORFs which encode highly charged basic proteins with repetitive motifs which may serve as regulatory molecules. The finding that the deduced peptides of two different reading frames showed sequence similarity suggests that ORF3 contains a translational frameshift which creates a molecular switch responsible for phase variation. The function of ORF3 is uncertain, but mutations could not be obtained, suggesting that it is essential for viability [7].

The variants do not change the physical characteristics of the peptidoglycan stem peptides, but opaque variants do not undergo autolysis as quickly as transparent variants. Although the expression of the gene encoding the major pneumococcal autolysin (lytA) is less in opaque variants [8], colonisation differences in the infant rat nasopharynx do not correlate with lytA expression, as $l y t A^{-}$strains were able to colonise and bind at the same rate as the $l y t A^{+}$parent. More importantly, opaque variants are resistant to the lytic effects of deoxycholate [7] and the addition of exogenous autolysin does not restore this defect, again supporting the idea that lytA expression is not the cause of the different phenotype. The opaque phenotype is associated with enhanced production of capsular polysaccharide and pneumococcal surface protein A
(PspA) [9] and the transparent phenotype with increased amounts of cell wall-associated teichoic acid (C-polysaccharide). The choline-binding protein CbpA is present in higher concentrations in transparent phenotypes and this protein is required for efficient colonisation in the infant rat model (see below) [10]. Opaque variants have been shown to be more virulent in the mouse intraperitoneal model of infection with a reduction of 2-3 days in the time to death [9]. Organisms of the same phenotype were recovered from the spleens of mice that died after inoculation with opaque phenotypes, whereas in those mice inoculated with a transparent phenotype, organisms of a more opaque phenotype were recovered from their spleens [9]. Table 1 summarises the differences between opaque and transparent phenotypes.

Attachment and phase variation. S. pneumoniae binds to oral epithelial cells via $\mathrm{N}$-acetyl galactosamine $\beta 1-3$ galactose $[11,12]$. Resting pneumocytes express two classes of receptor: $N$-acetyl-D-galactosamine with either $\beta 1,3$ or $\beta 1,4$ links to galactose. Transparent and opaque phenotypes bind equally well to these receptors. Activated type II pneumocytes express a PAF receptor when stimulated by cytokines such as IL-1. Transparent, but not opaque, colonial variants of pneumococci adhere to the PAF receptor via a phosphorylcholine ligand. It has been proposed that the pneumococcus binds to the PAF receptor by mimicking PAF, which, like the pneumococcal cell surface, contains phosphorylcholine. Transparent phenotypes harbour more phosphorylcholine-containing cell-wall teichoic acid components and binding is reduced by PAF receptor antagonists and exogenous choline [6]. The importance of cell-surface phosphorylcholine is illustrated by the finding that binding is

Table 1. Summary of differences between opaque and transparent phenotypes

\begin{tabular}{lcc}
\hline Characteristic & Transparent & Opaque \\
\hline Capsule production & + & +++ \\
Teichoic acid & ++++ & + \\
Surface expression $l y t A$ & +++ & + \\
PspA & + & ++ \\
Colonisation & +++ & + \\
Virulence & + & +++ \\
\hline
\end{tabular}

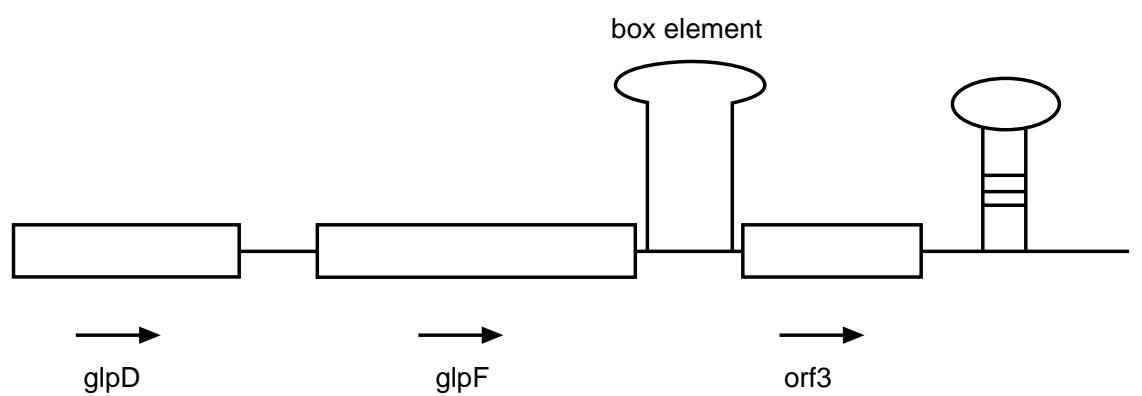

Fig. 1. Map of the opacity locus. The direction of translation is indicated by arrows. A putative transcriptional terminator is indicated after ORF3. (Redrawn from published data [7].) 
reduced 100-fold when pneumococci are grown in ethanolamine and express ethanolamine instead of phosphorylcholine [13].

Pneumococci cannot enter resting endothelial cells, but $2-3 \%$ of the inoculum is internalised by activated cells [14]. Activated endothelial cells upregulate PAF receptor expression and allow pneumococcal entry by the PAF receptor recycling pathway - this may provide a route of transition across the alveolar space into the bloodstream and across the blood-brain barrier into the meninges. It is thought that either viral infections or cell-wall components released from dividing pneumococci could provide the initial stimulus for cytokine production [15].

Pneumococcal surface adhesin A (PsaA) is a recently identified $37-\mathrm{kDa}$ surface lipoprotein essential for pneumococcal virulence. Sequencing studies have shown homology between PsaA and lipoprotein adhesins found in other viridans-type streptococci, such as FimA in $S$. parasanguis [16] and SsaB in $S$. sanguis [17]. PsaA is a component of an ABC-type manganese permease membrane transport system many such transporters exist in the pneumococcal genome and some, such as Adc and PsaA, are particularly important for virulence.

PsaA is encoded by the psaA gene, which is part of the psa locus. This locus contains three other ORFs encoding an adenosine triphosphate (ATP)-binding protein (PsaB), a hydrophobic membrane protein (PsaC) and another protein (PsaD) which has homology with a periplasmic thiol peroxidase of Escherichia coli.

In keeping with the actions of other pneumococcal permeases, the psa operon appears to have a regulatory role in adhesion. This operon affects the expression of choline-binding proteins on the pneumococcal surface, psa mutants demonstrating a complete absence of CbpA [18]. PsaA ${ }^{-}$and $\mathrm{PsaD}^{-}$mutants have been shown to display reduced virulence in mice and reduced adherence to lung cells in vitro [19]. $\mathrm{psaB}^{-}$, psa $\mathrm{C}^{-}$and $\mathrm{psaD}^{-}$mutants have been noted to have a reduced capacity for transformation. Mutations within the psa operon have also been shown to confer penicillin tolerance, mutants being able to survive $10 \times \mathrm{MIC}$ of penicillin [18].

Pneumococcal surface protein C. Pneumococcal surface protein C (Psp C) is a surface protein that crossreacts with Psp A but, unlike Psp A, is essential for pneumococcal carriage. Its C-terminal and proline-rich domains are very similar to those of Psp A; however, its $\alpha$-helical domain is $50-100 \%$ larger, containing an additional repeat of 100 amino acids [20].

Under different names (PbcA, SpsA, CBPA) several different properties have been attributed to the PspC molecule. Its abilities to bind $\mathrm{C} 3$ and secretory $\operatorname{IgA}$ were described under the names $\mathrm{PbcA}$ and $\mathrm{Sps} A$ respectively. The properties of this protein under its original name, CBPA, are discussed below.

Choline-binding protein $A(C b p A)$ was the first pneumococcal surface protein adhesin to be described [8]. It is composed of 663 amino acids and has a molecular mass of $75 \mathrm{kDa}$. The protein has two distinct domains - a C-terminal that shares $>95 \%$ homology with PspA and a unique $\mathrm{N}$-terminal which, although different in primary sequence, has an $\alpha$-helical coiled-coil structure like PspA. Like PspA, the choline-binding C-terminal of $\mathrm{CbpA}$ has a proline-rich region and the 10 tandem, direct repetitive sequences of 20 amino acids that is characteristic of choline-binding proteins. The structure of a choline-binding domain is illustrated in Fig. 2 [21].

$\mathrm{CbpA}$ is thought to play an important role in the adhesion of pneumococci to human cells - its expression is upregulated in the transparent phenotype and it has been shown that CbpA is essential for pneumococcal penetration across the blood brain barrier (see below) [22]. It has been suggested that pneumococci first adhere to cell surface glycoconjugates. Cytokine activation of the cells leads to a change in the expression of glycoprotein ligands, allowing increased pneumococcal adherence [23], and it is in this second stage - the change from colonisation to invasion - that CbpA has a function.

CbpA may act as a bridge between pneumococcal phosphorylcholine and activated human cell glycoconjugates; it could also mask phosphorylcholine by preventing it from binding to the PAF receptor. It is apparent that the adhesive/invasive process is a complex interplay between CbpA and phosphorylcholine, and their precise relationship and relative importance remain unclear.

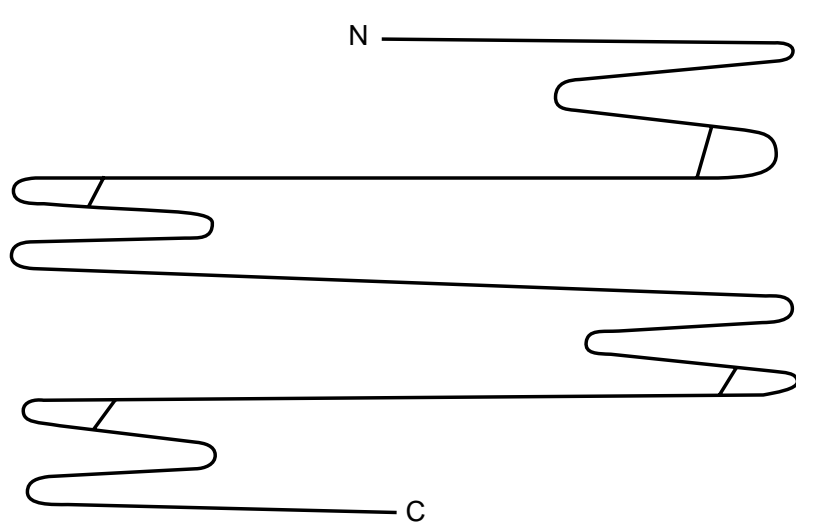

Fig. 2. Secondary structure of a hypothetical cholinebinding domain containing two tandem repeated units, showing $\beta$-turn structures. Choline produces a redistribution of the $\beta$-sheet and $\beta$-turn contributions. (Redrawn from published data [21].) 


\section{Invasion}

The most important event in pneumococcal infection is invasion of the lower respiratory tract; whilst pneumococcal pneumonia is in itself a life-threatening illness, it also serves as a focus for bloodstream invasion. Paradoxically, pneumococci also have an ability to establish systemic invasion in the absence of a clinically evident focus of infection. A recent study recorded this phenomenon in $15 \%$ of bacteraemias, most commonly in children [24].

At the alveolar level, the factors that determine whether or not invasive disease is established could be viewed as a dynamic balance between adherence and uptake into pneumocytes versus clearance by alveolar macrophages. Table 2 summarises the various scenarios and their likely outcomes.

The global population of pneumococci consists of $>90$ serotypes, but human infection/colonisation is dominated by a relatively small number of serotypes. Multilocus sequence typing has shown that some sequence types define strains with an increased capacity to cause invasive disease [25]. These apparently successful clones may have gathered a collection of genes (or have enhanced expression of a group of genes) that confer a selective advantage that shifts the host-pathogen balance, facilitating the transition from colonisation to invasion. Some of the proteins encoded by these genes are discussed below.

Hyaluronidase could facilitate pneumococcal invasion by degrading connective tissue. The importance of this enzyme has been illustrated by the demonstration that pneumococcal strains with higher hyaluronidase activity breach the blood-brain barrier and disseminate more effectively [26]. Studies have shown that pneumococcal strains isolated from patients with meningitis and meningoencephalitis have significantly higher hyaluronidase activity than strains causing purulent otitis media, indicating the importance of hyaluronidase in the pathogenesis of human pneumococcal meningitis [27].

Neuraminidase enzymes cleave sialic acid residues from a wide variety of molecules, damaging host tissues. This action may serve to expose receptors for pneumococcal adhesins, facilitating both colonisation and invasion. The role of neuraminidase in adhesion has been demonstrated recently in the chinchilla respiratory tract - neuraminidase treatment of the trachea increased pneumococcal adherence and reversed the inhibition produced by prior incubation with glycoconjugate analogues of known pneumococcal receptors. These data suggest that neuraminidase facilitates adhesion by increasing the number of adhesins available for pneumococcal binding [28].

There are two pneumococcal enzymes with neuraminidase activity, NanA and NanB, with very little aminoacid homology between them [29]. Although both are exported proteins with typical signal peptides, NanB lacks the typical cell-surface anchorage domain (LPXTGE) contained in NanA. NanA and NanB also have different predicted molecular masses, of $108 \mathrm{kDa}$ and $74.5 \mathrm{kDa}$ respectively, and widely different $\mathrm{pH}$ optima; this latter disparity might be of benefit in enabling the pneumococcus to adapt to different environmental conditions.

The importance of neuraminidase activity in pneumococcal virulence has been suggested by the finding that patients with pneumococcal meningitis with high levels of $\mathrm{N}$-acetyl neuraminic acid in their cerebrospinal fluid (CSF) were more likely to develop coma and bacteraemia [30].

Pneumococcal surface protein $A(P s p A)$ has been confirmed as a pneumococcal virulence determinant. Immunisation of X-linked immunodeficient mice with recombinant PspA conferred protective immunity to challenge with three of four virulent pneumococcal strains [31], and monoclonal anti-PspA provided passive protection [32].

PspA is an $84-\mathrm{kDa}$ protein comprising several distinct domains [33]. From the amino-acid sequence of the Nterminal end, an $\alpha$-helical coiled-coil structure has been predicted [34]. The $\alpha$-helices contain a patchwork of conserved and variable areas. The sequence of the first 45 amino acids of the N-terminal domain has a 7residue periodicity common to fibrous proteins such as streptococcal M-protein. Adjacent to the $\alpha$-helical domain is a proline-rich domain containing a predominant sequence (pro-ala-pro-ala). The C-terminal

Table 2. Likelihood of pneumococcal pulmonary invasion based on different alveolar conditions

\begin{tabular}{llll}
\hline Situation & $\begin{array}{l}\text { Pneumocyte } \\
\text { uptake }\end{array}$ & $\begin{array}{l}\text { Macrophage } \\
\text { clearance }\end{array}$ & Outcome \\
\hline $\begin{array}{l}\text { Resting pneumocytes, } \\
\text { opsonic antibody absent }\end{array}$ & Low & Low & Low risk of invasion \\
$\begin{array}{l}\text { Resting pneumocytes, } \\
\text { opsonic antibody present } \\
\begin{array}{l}\text { Activated pneumocytes, } \\
\text { opsonic antibody absent }\end{array}\end{array}$ & Low & High & Very low risk of invasion \\
$\begin{array}{l}\text { Activated pneumocytes, } \\
\text { opsonic antibody present }\end{array}$ & High & Low & Invasion likely \\
\hline
\end{tabular}


end of PspA is the choline-binding component, made up of 10 repeats of 20 amino acids each. This region is homologous to other pneumococcal choline-binding proteins, and is responsible for the attachment of PspA to the pneumococcal surface [35]. Adjacent to this region is a hydrophilic domain of 17 amino acids, the function of which is unclear.

Recent work has shown that Psp A functions as a specific receptor for lactoferrin, the $\mathrm{N}$-terminal being responsible for lactoferrin binding [36]. Hence, Psp A plays an essential role in enabling iron acquisition by $S$. pneumoniae. Mutant strains lacking PspA expression fix more complement than isogenic parent strains expressing PspA [16] - this interference with complement activation serves to facilitate pneumococcal survival and host invasion.

Pneumolysin. There has been considerable progress in the characterisation of pneumolysin. The importance of pneumolysin as a virulence determinant has been recognised following active and passive immunisation studies of mice [37]. This finding has since been confirmed by studies demonstrating considerable loss of virulence in isogenic mutants of $S$. pneumoniae in which the gene for pneumolysin had been disrupted [38].

Pneumolysin is a cytoplasmic toxin released by autolysis of the cell. It belongs to the family of thiolactivated cytolysins, the mechanism of action of which is thought to follow a two-stage common pathway [39]. The first stage entails binding of the monomeric toxin to the target cell membrane. The second stage comprises lateral movement and oligomerisation of the monomers to form a high mol. wt transmembrane pore. This results in leakage of intracellular solutes and an influx of water, resulting in colloid-osmotic lysis of the cell. By this mechanism, pneumolysin is able to damage a wide range of eukaryotic cells. Pneumolysin also has modulatory effects on cells at sublytic concentrations, e.g., stimulation of TNF- $\alpha$ and IL- $1 \beta$ production by human monocytes [40].

Pneumolysin is a $52.8-\mathrm{kDa}$ polypeptide consisting of 470 amino acids. Whilst much of its structure/function relationship remains unclear, the functional relevance of some areas has been elucidated. Pneumolysin contains eight histidine residues, one of which (position 367 in pneumolysin) is conserved in all thiol-activated toxins. Mutagenesis of this residue seriously impairs oligomerisation, causing a dramatic loss of activity [41]. Also in common with other thiol-activated toxins, pneumolysin contains only a single cysteine residue (at position 428 in a domain towards the carboxyl terminus). Substitution of this residue has been shown to greatly reduce cytolytic activity without affecting binding or oligomerisation [42], implying that there is a crucial action required for target cell lysis beyond oligomerisation, in which this residue plays an essential role. Deletion studies have shown that the C-terminus is involved in membrane binding [43] via cholesterol [44].

The secondary structure of pneumolysin consists of $36 \% \beta$-sheet and 31\% $\alpha$-helix [45]. Hydropathy plots suggest that it is a highly hydrophilic molecule with no significant regions of hydrophobicity; hence, oligomerisation is essential for the formation of channels. Electron micrographs show that pneumolysin monomers are asymmetric molecules composed of four domains [46]. Three of the domains lie side by side, forming the stalk of the oligomer, from which domain 4 projects out as a flexible flange.

Oligomeric pneumolysin exists as large arc and ring structures measuring $30-40 \mathrm{~nm}$ in diameter in cell membranes [47]. The problem posed by this is the apparent absence of hydrophobic areas on both the surface of pneumolysin and at the domain interfaces making membrane insertion impossible. However, there is a hydrophobic pocket that could act as a cholesterolbinding site. This pocket is blocked by a tryptophan-rich region at the tip of domain 4, of which one residue (Trp 433 ) is crucial in maintaining a loop conformation; it is hypothesised that cholesterol could displace the tryptophan-rich region to reveal the hydrophobic pocket which could then initiate membrane penetration [48].

The cytolytic properties of pneumolysin enable it to inflict damage on a wide variety of eukaryotic cells, including bronchial epithelial cells, alveolar epithelial cells and pulmonary endothelium. Its detrimental effects on bronchial epithelium result in slowing of the ciliary beat, impairing the ability of the mucociliary escalator to clear particles effectively. Pneumolysin also induces separation of the tight junctions of the alveolar epithelial cells [49]. This, together with its direct cytotoxic effect on the cells of the alveolar capillary barrier, serve to facilitate bloodstream invasion by pneumococci. Pneumolysin also inhibits the respiratory burst and chemotaxis of polymorphs at concentrations as low as $1 \mathrm{ng} / \mathrm{ml}$ [50], hence facilitating pneumococcal survival and invasion.

Opsonophagocytosis by the complement system is essential for clearance of pneumococci from the lung [51]. The ability of pneumolysin to activate the classical complement pathway may allow pneumococci to evade opsonophagocytosis by consuming the limited supply of complement factors in the alveoli, facilitating invasion. The effectiveness of the complement-activating property of pneumolysin in reducing pneumococcal clearance and increasing mortality has been demonstrated recently in rats [52].

\section{Inflammation and shock}

Pneumolysin. The mechanism by which pneumolysin activates the classical complement cascade remains 
controversial. It has been thought that its ability to do this might be related to sequence similarity between pneumolysin and C-reactive protein (CRP), a human acute phase reactant capable of activating the classical complement cascade by binding to $\mathrm{C} 1 \mathrm{q}$. However, molecular structural studies have shown that although there is sequence similarity between the two, this does not translate into structural homology and, therefore, an alternative mechanism must exist. Although domain 4 of pneumolysin and IgG Fc fragment have little sequence similarity, they both adopt a $\beta$ sandwich structure; this raises the possibility that structural similarity between aggregated $\mathrm{IgG}$ and domain 4 of oligomeric pneumolysin leads to pneumolysin-induced activation of the classical complement pathway.

An interesting mechanism of injury that mediates both the activation of the inflammatory response and direct lung damage is suggested by the finding that phospholipase A in pulmonary artery endothelium is strongly activated by pneumolysin, and once activated breaks down a wide variety of cell-membrane phospholipids [53]. This results in the release of free fatty acids and lysophosphatides, which are directly cytotoxic. Arachidonic acid released by phospholipase activity, together with its eicosanoid cascade metabolites, are potent neutrophil chemotaxins. Recruitment and activation of neutrophils further contribute to lung damage, as well as to the inflammatory response. Inflammation would be enhanced by the ability of pneumolysin to activate the classical complement pathway without the need for specific antibody [54].

Pneumolysin plays an important role as a general inflammatory agonist. It functions as a TNF- $\alpha$ agonist and induces expression of cyclo-oxygenase- 2 , TNF- $\alpha$ and IL-6. Pneumolysin is also the main inducer of nitric oxide (NO) production in macrophages [55].

Autolysin. The major enzyme responsible for cell-wall turnover is the autolysin of $S$. pneumoniae, $N$-acetyl muramoyl-L-alanine amidase. This enzyme is responsible for cellular autolysis which occurs at the end of $\log$ phase in pneumococci, and lysis of the cell wall in response to treatment with deoxycholate or with $\beta$ lactam antibiotics [56]. Autolysin is one of about a dozen proteins that are held on the pneumococcal surface by docking non-covalently with the choline of the pneumococcal cell wall, instead of the usual method of anchorage in gram-positive bacteria using the LPXTGE motif. These choline-binding proteins have a common choline-binding carboxy-terminal, their functional diversity being produced by the aminoterminal.

Analytical centrifugation experiments indicate that autolysin forms a tail-to-tail dimer through interaction between the choline-binding domains. This dimer is strongly stabilised during choline binding, forming a prolate ellipsoid with a catalytic domain exposed at each end [57]. The COOH-domain is constructed of six repeated units, and binds choline phosphate residues of teichoic acid. It is thought that in this position the catalytic amidase domain, located in the N-terminus, is placed in an optimal position to degrade peptidoglycan [58]. The shape of this molecule would facilitate its diffusion through the cross-linked framework of the cell wall and increase the number of bonds available for hydrolysis. It may also facilitate motion of the enzyme along the septum by a stepwise binding and release mechanism [57]. The molecule appears to possess two choline-binding sites, one of low and one of high affinity.

The secondary structure of autolysin contains $19 \% \alpha-$ helix, $47 \% \beta$-sheet, $23 \%$ turns and $11 \%$ irregular structures [59]. The far UV spectrum of the protein is governed by chiral contributions from aromatic residues clustered in the $\mathrm{COOH}$ domain. Similar results have been obtained for other choline-binding proteins such as Cpl 1 lysozyme [60]. The addition of choline modifies the far UV spectrum by alteration of the environment of the aromatic clusters, but the secondary structure remains unaltered. Dimerisation appears to occur through co-operation between the low-affinity choline binding sites [59].

Although the sequence of lytA has been known for some time, the degree of allelic variation between isolates remains contentious. Although single-strand conformational polymorphism analysis suggested considerable variation both among clinical isolates and between clinical isolates and the published sequence [61], more recent restriction fragment length polymorphism and nucleotide sequencing studies suggest that $l y t A$ is highly conserved, displaying limited genetic variation $(0.11-3.2 \%)$ [62].

Autolysin contributes to pneumococcal virulence in several ways. Its role in cell-wall turnover means that autolysin activity generates cell-wall breakdown products, which are highly inflammatory. The cell-walldegrading activity of autolysin also allows the release of intracellular toxins (e.g., pneumolysin) into the external medium. Several experimental studies have demonstrated the role of autolysin in virulence. Reduced virulence has been demonstrated when normally virulent strains are transformed with inactivated lytA and the deficit has been shown to be reversible by back transformation [63].

C-polysaccharide. The cell wall of $S$. pneumoniae has an important role in generating inflammatory mediators and consequent pathological changes. Injection of cellwall preparations, but not heat-killed capsulate strains or isolated capsular antigen, has been shown to generate a strong inflammatory response in chinchilla rabbits [64]. The teichoic acid C-polysaccharide (PnC) is important, as removal of this antigen diminishes 
complement activation by cell-wall components [65]. The components of the complement cascade generated by interaction with $\mathrm{PnC}$ are thought to be crucial in generation of an inflammatory reaction in the alveoli [66], the meninges [64] and the middle ear. PnC activates the alternate complement pathway via its phosphorylcholine (PC) component $[67,68]$. The pneumococcal plasma membrane is also a weak activator of the alternate pathway through the PnC analogue lipoteichoic acid ( $F$ antigen) [68]. In the chinchilla model of otitis media, death of the organism induced by penicillin was responsible for a rapid increase in the inflammatory response. This was thought to be due to the release of cell-wall products and pneumolysin [69]. Organisms with high cell-wall turnover, resulting in a greater release of teichoic acid components, induce a greater inflammatory response in the rabbit meninges [70].

There are reports of the importance of circulating PnC antigen in naturally acquired human infection. Coonrod and Leach described the detection of C-polysaccharide antigen in the serum of a splenectomised patient who had died of fulminant bacteraemia [71]. In a clinical study, a trend to higher mortality was found in patients with detectable $\mathrm{PnC}$ in their serum [72]. There are many reports of the protective effect of the administration of anti-PC antibodies or CRP, which also binds C-polysaccharide via $\mathrm{PC}$, when given to mice challenged with a fatal pneumococcal infection $[73,74]$. Conversely, there are reports in which anti-PC antibody failed to provide a protective effect [73]. These data must be considered along with the evidence of the protective efficacy of vaccines that stimulate anti-PC antibodies [75]. Antibody to PC is not opsonic and so cannot act in the same way as anti-capsular antibody - it may provide protection by reducing the impact of the release of inflammatory C-polysaccharide antigens.

PC is of considerable functional relevance in organisms colonising mucosal surfaces, particularly the respiratory tract. The expression of this epitope is subject to phase-variation, there being a selection for $\mathrm{PC}^{+}$ variants during nasopharyngeal colonisation. PC has been shown to protect organisms against clearance, hence contributing to persistence in the respiratory tract. However, $\mathrm{PC}^{+}$variants exhibit increased serum sensitivity. Thus, it appears that there is differential expression of PC, with up-regulation occurring in the initial colonisation phase and down-regulation in the subsequent invasive phase in order to avoid anti-PC antibody, CRP binding and complement activation [76].

\section{Immunopathology}

Immune response. The humoral and cellular arms of the immune system act in concert to defend the host against pneumococcal infection. The major immune mechanism protecting the host against pneumococcal infection is opsonophagocytosis [77]. Opsonisation is initiated by antibodies reactive against capsular polysaccharides, but is also complement dependent, optimal pneumococcal clearance requiring intact classical and alternate pathways. CRP complexes with pneumococcal C-polysaccharide and also possesses some opsonic activity; however, the protective effects of CRP against pneumococcal infection are mediated mainly via activation of the classical complement pathway [78]. Comprehensive reviews on this complex subject have been published previously.

Pneumonia. The interactions that occur between the pneumococcus and host during the evolution of infection are complex and remain to be fully elucidated. In a mouse model, pulmonary pneumococcal infection has broadly been divided into five stages [79]. In the first stage $(0-4 \mathrm{~h})$, infection is established - there is evidence of ineffective phagocytosis by alveolar macrophages and cytokine release. The major pneumococcal antiphagocytic components are its polysaccharide capsule and pneumolysin (see above). The predominant cytokines vary between sites, with TNF, IL-6 and NO predominating in bronchial lavage (BAL) fluid; TNF, IL-6 and interleukin-1 (IL-1) in lung tissue; and IL-6 in serum. The second stage (4-24 h) is marked by the multiplication of pneumococci in the alveoli and neutrophil chemotaxis into lung tissue TNF, IL-6, IL-1 and leukotriene $\mathrm{B}_{4}$ levels all increase in both lung tissue and BAL fluid. Serum IL-1 levels also rise transiently. Neutrophil chemotaxis is mediated by the complement-activating properties of pneumolysin and C-polysaccharide as well as the cytokines listed above. Lung damage is seen in the third stage (24$48 \mathrm{~h}$ ) - there is evidence of alveolar injury and interstitial oedema - reflecting the cytolytic actions of pneumolysin. There is also evidence of regeneration during this stage - type II pneumocytes proliferate to regenerate both type I and type II cells. This stage sees the progression of infection from the pre-septicaemic to the septicaemic phase, bacteria shifting from the alveoli through lung tissue into the bloodstream and a reduction in IL-1 and TNF in the lungs. Both CbpA and the changes made to the alveolar-capillary barrier by pneumolysin (see above) would facilitate bloodstream invasion. The fourth stage $(48-72 \mathrm{~h})$ is characterised by a sharp increase in alveolar monocyte and lymphocyte activity, together with NO release in lung tissue and alveolar spaces. The effects of pneumococcal bacteraemia are manifest - leucopenia and thrombocytopenia are seen, together with an increase in blood TNF and IL-6. The final stage (72$96 \mathrm{~h}$ ) is characterised by further bacterial proliferation, high NO levels, massive tissue damage, lipid peroxidation and high mortality.

Cytokine production is stimulated by both CD14dependent and -independent pathways, with as many as two receptors other than CD14 being involved [80]. Although the precise roles and effects of the various 
cytokines produced at the different stages of the pathogenesis of pneumococcal pneumonia remain to be fully elucidated, some progress has been made towards unravelling this maze.

There is considerable evidence to suggest that IL-1 plays a far more dominant role in gram-positive inflammation than it does in gram-negative sepsis [81]. Recent work indicates that TNF, although pivotal in the pathogenesis of gram-negative sepsis syndrome, plays a protective role in pneumococcal pneumonia, inhibiting the onset of bacteraemia by stimulating antibody production, neutrophil recruitment and granulocyte-macrophage colony-stimulating factor production by endothelial cells [82]. IL-10, on the other hand, demonstrates a protective role in gram-negative sepsis, but elevated lung levels have been shown to be detrimental in a murine model of pneumococcal pneumonia [83]. There is also evidence that IL-6 plays an important role as a mediator of inflammation, controlling the production of both pro- and antiinflammatory cytokines; however, its net effect has been shown to be protective [84].

Meningitis. There has been considerable recent advance in our understanding of pneumococcal meningitis. Whether meningeal invasion is the result of specific targeting or is purely a chance process remains unclear. However, pneumococci have been shown to be nearly twice as efficient at invading cerebral as compared with peripheral endothelium. Transparent phenotypes, with less capsular polysaccharide, more teichoic acid and more $\mathrm{CbpA}$, are three-to-five times more efficient at invading cerebral microvascular endothelia than are opaque phenotypes, which are killed intracellularly. This is in contrast to the bloodstream, where opaque phenotypes are more invasive. Inflammatory activation is a prerequisite for the migration of pneumococci through cerebral microvascular endothelial cells. It is thought that transparent phenotypes are able to enter by PAF receptor-mediated endocytosis and corrupt the PAF receptor recycling pathway, producing a greater degree of vesicular transcytosis to the basolateral surface of the cell, with some recycling to the apical surface. Opaque variants, in contrast, appear to be killed intracellularly [22].

It has been shown that only a very low inoculum of capsulate pneumococci is required to establish infection in a rabbit model [85]. S. pneumoniae does not directly mediate cytotoxicity. Rather, tissue damage is the result of inflammation and its sequelae. The critical stimuli for the inflammatory response are the major components of the pneumococcal cell wall (teichoic acid and peptidoglycan) and pneumolysin, and not the polysaccharide capsule, which lacks inflammatory potential and acts mainly to protect pneumococci against phagocytosis in the absence of anticapsular antibody $[64,86]$. Although different inflammatory responses have been found with strains of different capsular serotypes $[87,88]$, the strains studied were not otherwise isogenic and, therefore, it is not possible to ascribe these differences to any one parameter. Cellwall components released by autolytic activity induce release of pro-inflammatory cytokines (e.g., TNF- $\alpha$, IL1, IL-6) from mononuclear cells within the central nervous system (CNS) such as ependymal cells, astrocytes and macrophages/microglia. These cytokines trigger a complex cascade of inflammatory mediators, which in concert regulate the various arms of the inflammatory response [89].

The cytokines released result in an opening of the tight junctions between brain capillary endothelial cells associated with enhanced pinocytotic activity, allowing an influx of serum components, notably chemotactic complement factors [90,91]. They also upregulate adhesion molecules (e.g., ICAM-1) on cerebral vascular endothelial cells and attract neutrophils across the blood-brain barrier [92]. In consequence, a neutrophil infiltrate is evident in the CSF within $8-12 \mathrm{~h}$ of bacterial inoculation [93]. However, the neutrophils have no effect on pneumococcal multiplication, reflecting the low concentration of opsonins in the CSF [9496].

The cytokine milieu may also have more direct neurotoxic effects by contributing to a metabolic shift to anaerobic glycolysis, astrocyte dysfunction and neuronal damage [92].

Probably the most important consequence of this inflammatory reaction in the CSF is increased intracranial pressure, which results from cerebral oedema, increased cerebral blood volume and alterations of CSF hydrodynamics (in particular reduced CSF resorption) [97-100]. This increased intracranial pressure, together with the impairment of autoregulation of cerebral blood flow seen in pneumococcal meningitis, plays a significant role in reducing cerebral blood flow, resulting in ischaemic necrosis and neuronal loss from energy failure. Several vasoconstrictive mediators, such as endothelin and reactive oxygen intermediates, counteract the vasodilatory effects of the increased NO production seen in meningitis and further contribute to cerebral ischaemia. These agents also have a variety of deleterious effects on cells and macromolecules including lipid peroxidation, DNA damage and protein oxidation [93].

Direct neurotoxicity is another important mechanism by which brain damage occurs. It is mediated by pneumolysin and several agents released in pneumococcal meningitis. NO can be directly neurotoxic or can combine with superoxide to form peroxynitrite, a highly cytotoxic agent [93]. The production of NO by neuronal nitric oxide synthase (NOS) and oxidative radical release is increased by excitatory amino acids (e.g., glutamate), which are released at higher levels from neurones under stress. A commonly affected site 
is the dentate gyrus in the hippocampus, cell death occurring by apoptosis [92].

\section{Concluding remarks}

In previous reviews, the various antigens and phenomena have been presented. The present paper aimed to describe the way in which pneumococci perturb lung defences, attach and invade to cause severe disease. As new information becomes available, a better understanding of these critical processes will be developed and this will enable clinicians to intervene more effectively and reduce the continuing high mortality exacted by this versatile pathogen.

\section{References}

1. James JF, Swanson J. Studies on gonococcus infection XIII. Occurrence of color/opacity colonial variants in clinical cultures. Infect Immun 1978; 19: 332-340.

2. Weiser JN. Relationship between colony morphology and the life cycle of Haemophilus influenzae: the contribution of lipopolysaccharide phase variation to pathogenesis. J Infect Dis 1993; 168: 672-680.

3. Paul JR. Pneumococcus variants. I. Intermediate forms and the influence of environment in their production during invitro $\mathrm{S}$ to $\mathrm{R}$ and $\mathrm{R}$ to $\mathrm{S}$ transitions. J Bacteriol 1934; 28: 45-68.

4. Weiser JN, Austrian R, Sreenivasan PK, Masure HR. Phase variation in pneumococcal opacity: relationship between colonial morphology and nasopharyngeal colonization. Infect Immun 1994; 62: 2582-2589.

5. Cundell DR, Weiser JN, Shen J, Young A, Tuomanen EI. Relationship between colonial morphology and adherence of Streptococcus pneumoniae. Infect Immun 1995; 63: 757-761.

6. Cundell DR, Gerard NP, Gerard C, Idanpaan-Heikkila I, Tuomanen EI. Streptococcus pneumoniae anchor to activated human cells by the receptor for platelet-activating factor. Nature 1995; 377: 435-438.

7. Saluja SK, Weiser JN. The genetic basis of colony opacity in Streptococcus pneumoniae: evidence for the effect of box elements on the frequency of phenotypic variation. Mol Microbiol 1995; 16: 215-227.

8. Weiser JN, Markiewicz Z, Tuomanen EI, Wani JH. Relationship between phase variation in colony morphology, interstrain variation in cell wall physiology and nasopharyngeal colonization by Streptococcus pneumoniae. Infect Immun 1996; 64: 2240-2245.

9. Kim JO, Weiser JN. Association of intrastrain phase variation in quantity of capsular polysaccharide and teichoic acid with the virulence of Streptococcus pneumoniae. J Infect Dis 1998; 177: $368-377$.

10. Rosenow C, Ryan P, Weiser JN et al. Contribution of novel choline-binding proteins to adherence, colonization and immunogenicity of Streptococcus pneumoniae. Mol Microbiol 1997; 25: 819-829.

11. Andersson B, Dahmén T, Freijd T et al. Identification of an active disaccharide unit of a glycoconjugate receptor of pneumococci attaching to human pharyngeal epithelial cells. $J$ Exp Med 1983; 158: 559-570.

12. Andersson B, Beachey EH, Tomasz A, Tuomanen E, Svanborg-Edén C. A sandwich adhesion on Streptococcus pneumoniae attaching to human oropharyngeal epithelial cells in vitro. Microb Pathog 1988; 4: 267-278.

13. Cabellos C, MacIntyre DE, Forrest M, Burroughs M, Prasad $\mathrm{S}$, Tuomanen E. Differing roles for platelet-activating factor during inflammation of the lung and the subarachnoid space. The special case of Streptococcus pneumoniae. J Clin Invest 1992; 90: 612-618.

14. Geelen S, Bhattacharyya C, Tuomanen E. The cell wall mediates pneumococcal attachment to and cytopathology in human endothelial cells. Infect Immun 1993; 61: 1538-1543.
15. Tuomanen E. Molecular and cellular biology of pneumococcal infection. Curr Opin Microbiol 1999; 2: 35-39.

16. Fenno JC, LeBlanc DJ, Fives-Taylor P. Nucleotide sequence analysis of a type 1 fimbrial gene of Streptococcus sanguis FW213. Infect Immun 1989; 57: 3527-3533.

17. Ganeshkumar N, Hannam PM, Kolenbrander PE, McBride BC. Nucleotide sequence of a gene coding for a salivabinding protein (SsaB) from Streptococcus sanguis 12 and possible role of the protein in coaggregation with actinomyces. Infect Immun 1991; 59: 1093-1099.

18. Novak R, Braun JS, Charpentier E, Tuomanen E. Penicillin tolerance genes of Streptococcus pneumoniae: the ABC-type manganese permease complex Psa. Mol Microbiol 1998; 29: 1285-1296.

19. Berry AM, Paton JC. Sequence heterogeneity of PsaA, a 37kilodalton putative adhesin essential for virulence of Streptococcus pneumoniae. Infect Immun 1996; 64: 5255-5262.

20. Briles DE, Hollingshead SK, Swialto E et al. PspA and PspC: their potential for use as pneumococcal vaccines. Microb Drug Resist 1997; 3: 401-408.

21. García JL, Sanchez-Beato AR, Medrano FJ, Lopez R. Versatility of choline-binding domain. Microb Drug Resist 1998; 4: 25-36.

22. Ring A, Weiser JN, Tuomanen EL. Pneumococcal trafficking across the blood brain barrier. Molecular analysis of a novel bidirectional pathway. J Clin Invest 1998; 102: 347-360.

23. Cundell DR, Masure HR, Tuomanen EI. The molecular basis of pneumococcal infection. A hypothesis. Clin Infect Dis 1995; 21 Suppl 3: S204-S212.

24. Balakrishnan I, Crook P, Morris R, Gillespie SH. Early predictors of mortality in pneumococcal bacteraemia. $J$ Infect 2000 40: 256-261.

25. Enright MC, Spratt BG. A multilocus sequence typing scheme for Streptococcus pneumoniae: identification of clones associated with serious invasive disease. Microbiology 1998; 144: 3049-3060.

26. Kostiukova NN, Volkova MO, Ivanova VV, Kvetnaya AS. A study of pathogenic factors of Streptococcus pneumoniae strains causing meningitis. FEMS Immunol Med Microbiol 1995; 10: 133-137.

27. Volkova MO, Kostiukova NN, Kvetnaia AS. [The role of hyaluronidase in the occurrence of a generalized pneumococcal infection.] Zh Mikrobiol Epidemiol Immunobiol 1994; Aug-Sept Suppl 1: 118-122 [in Russian].

28. Tong HH, McIver MA, Fisher LM, DeMaria TF. Effect of lacto-N-neotetraose, asialoganglioside-GM1 and neuraminidase on adherence of otitis media-associated serotypes of Streptococcus pneumoniae to chinchilla tracheal epithelium. Microb Pathog 1999; 26: 111-119.

29. Berry AM, Lock RA, Paton JC. Cloning and characterization of nanB, a second Streptococcus pneumoniae neuraminidase gene, and purification of the NanB enzyme from recombinant Escherichia coli. J Bacteriol 1996; 178: 4854-4860.

30. O'Toole RD, Goode L, Howe C. Neuraminidase activity in bacterial meningitis. J Clin Invest 1971; 50: 979-985.

31. McDaniel LS, Sheffield JS, Delucchi P, Briles DE. PspA, a surface protein of Streptococcus pneumoniae, is capable of eliciting protection against pneumococci of more than one capsular type. Infect Immun 1991; 59: 222-228.

32. McDaniel LS, Scott G, Kearney JF, Briles DE. Monoclonal antibodies against protease-sensitive pneumococcal antigens can protect mice from fatal infection with Streptococcus pneumoniae. J Exp Med 1984; 160: 386-397.

33. Yother J, Briles DE. Structural properties and evolutionary relationships of $\mathrm{PspA}$, a surface protein of Streptococcus pneumoniae, as revealed by sequence analysis. $J$ Bacteriol 1992; 174: 601-609.

34. Talkington DF, Brown BG, Thape JA, Koenig A, Russell H. Protection of mice against fatal pneumococcal challenge by immunization with pneumococcal surface adhesin A (PsaA). Microb Pathog 1996; 21: 17-22.

35. Yother J, Handsome GL, Briles DE. Truncated forms of PspA that are secreted from Streptococcus pneumoniae and their use in functional studies and cloning of the $p s p A$ gene. $J$ Bacteriol 1992; 174: 610-618.

36. Hammerschmidt S, Bethe G, Remane PH, Chhatwal GS. Identification of pneumococcal surface protein $A$ as a lactoferrin-binding protein of Streptococcus pneumoniae. Infect Immun 1999; 67: 1683-1687. 
37. Paton JC, Lock RA, Hansman DJ. Effect of immunization with pneumolysin on survival time of mice challenged with Streptococcus pneumoniae.Infect Immun 1983; 40: 548-552.

38. Berry AM, Yother J, Briles DE, Hansman D, Paton JC. Reduced virulence of a defined pneumolysin-negative mutant of Streptococcus pneumoniae. Infect Immun 1989; 57: 2037-2042.

39. Bhakdi S, Tranum-Jensen J. Membrane damage by poreforming bacterial cytolysins. Microb Pathog 1986; 1: 5-14.

40. Houldsworth S, Andrew PW, Mitchell TJ. Pneumolysin stimulates production of tumor necrosis factor alpha and interleukin-1 beta by human mononuclear phagocytes. Infect Immun 1994; 62: 1501-1503.

41. Mitchell TJ, Andrew PW, Boulnois GJ, Lee C-J, Lock RA, Paton JC. Molecular studies of pneumolysin as an aid to vaccine design. Zentralbl Bakteriol 1992; 23: 429-438.

42. Saunders FK, Mitchell TJ, Walker JA, Andrew PW, Boulnois GJ. Pneumolysin, the thiol-activated toxin of Streptococcus pneumoniae, does not require a thiol group for in vitro activity. Infect Immun 1989; 57: 2547-2552.

43. Owen RHG, Boulnois GJ, Andrew PW, Mitchell TJ. A role in cell-binding for the C-terminus of pneumolysin, the thiolactivated toxin of Streptococcus pneumoniae. FEMS Microbiol Lett 1994; 121: 217-222.

44. Alouf JE, Geoffroy C. The family of the antigenically-related cholesterol-binding ('sulphydryl activated') cytolytic toxins. In: Alouf JE, Freer JH (eds) Source of bacterial protein toxins. London, Academic Press. 1991: 147-186.

45. Morgan PJ, Varley PG, Rowe AJ, Andrew PW, Mitchell TJ Characterization of the solution properties and conformation of pneumolysin, the membrane-damaging toxin of Streptococcus pneumoniae. Biochem J 1993; 296: 671-674.

46. Morgan PJ, Hyman SC, Byron O, Andrew PW, Mitchell TJ, Rowe AJ. Modeling the bacterial protein toxin, pneumolysin, in its monomeric and oligomeric form. J Biol Chem 1994; 269: 25315-25320.

47. Morgan PJ, Hyman SC, Rowe AJ, Mitchell TJ, Andrew PW, Saibil HR. Subunit organisation and symmetry of poreforming, oligomeric pneumolysin. FEBS Lett 1995; 371: $77-80$.

48. Rossjohn J, Gilbert RJC, Crane D et al. The molecular mechanism of pneumolysin, a virulence factor from Streptococcus pneumoniae. J Mol Biol 1998; 284: 449-461.

49. Rayner CFJ, Jackson AD, Rutman A et al. Interaction of pneumolysin-sufficient and -deficient isogenic variants of Streptococcus pneumoniae with human respiratory mucosa. Infect Immun 1995; 63: 442-447.

50. Paton JC, Ferrante A. Inhibition of human polymorphonuclear leukocyte respiratory burst, bactericidal activity and migration by pneumolysin. Infect Immun 1983; 41: 1212-1216.

51. Winkelstein JA. Complement and the host's defence against the pneumococcus. Crit Rev Microbiol 1984; 11 187-208.

52. Alcantara RB, Preheim LC, Gentry MJ. Role of pneumolysin's complement-activating activity during pneumococcal bacteraemia in cirrhotic rats. Infect Immun 1999; 67: $2862-2866$.

53. Rubins JB, Mitchell TJ, Andrews PW, Niewoehner DE. Pneumolysin activates phospholipase A in pulmonary artery endothelial cells. Infect Immun 1994; 62: 3829-3836.

54. Paton JC, Rowan-Kelly B, Ferrante A. Activation of human complement by the pneumococcal toxin pneumolysin. Infect Immun 1984; 43: 1085-1087.

55. Braun JS, Novak R, Gao G, Murray PJ, Shenep JL. Pneumolysin, a protein toxin of Streptococcus pneumoniae, induces nitric oxide production from macrophages. Infect Immun 1999; 67: 3750-3756.

56. Tuomanen W, Tomasz A. Mechanism of phenotypic tolerance of nongrowing pneumococci to beta-lactam antibiotics. Scand J Infect Dis Suppl 1990; 74: 102-112.

57. Usobiaga $\mathrm{P}$, Medrano FJ, Gasset $\mathrm{M}$ et al. Structural organization of the major autolysin from Streptococcus pneumoniae. J Biol Chem 1996; 271: 6832-6838.

58. Markiewicz Z, Tomasz A. Protein-bound choline is released from the pneumococcal autolytic enzyme during adsorption of the enzyme to cell wall particles. J Bacteriol 1990; 172: 2241-2244.

59. Medrano FJ, Gasset M, López-Zúmel, Usobiaga P, Garcia L, Menéndez M. Structural characterization of the unligated choline-bound forms of the major pneumococcal autolysin
LytA amidase. $J$ Biol Chem 1996; 271: 29152-29161.

60. Sanz JM, Garcia JL. Structural studies of the lysozyme coded by the pneumococcal phage $\mathrm{Cp}-1$. Conformation changes induced by choline. Eur $J$ Biochem 1990; 187: 409-416.

61. Gillespie SH, McHugh TD, Ayres H, Dickens A, Efstratiou A, Whiting GC. Allelic variation in Streptococcus pneumoniae autolysin ( $N$-acetyl muramoyl- $L$-alanine amidase). Infect Immun 1997; 65: 3936-3938.

62. Whatmore AM, Dowson CG. The autolysin-encoding gene lytA of Streptococcus pneumoniae displays restricted allelic variation despite localized recombination events with genes of pneumococcal bacteriophage encoding cell wall lytic enzymes. Infect Immun 1999; 67: 4551-4556.

63. Berry AM, Lock RA, Hansman D, Paton JC. Contribution of autolysin to virulence of Streptococcus pneumoniae. Infect Immun 1989; 57: 2324-2330.

64. Tuomanen E, Liu H, Hengstler B, Zak O, Tomasz A. The induction of meningeal inflammation by components of the pneumococcal cell wall. J Infect Dis 1985; 151: 859-868.

65. Tomasz A, Saukkonen K. The nature of cell wall-derived inflammatory components of pneumococci. Pediatr Infect Dis $J$ 1989; 8: 902-903.

66. Tuomanen E, Rich R, Zak O. Induction of pulmonary inflammation by components of the pneumococcal cell surface. Am Rev Respir Dis 1987; 135: 869-874.

67. Winkelstein JA, Abramovitz AS, Tomasz A. Activation of C3 via the alternative complement pathway results in fixation of $\mathrm{C} 3 \mathrm{~b}$ to the pneumococcal cell wall. J Immunol 1980; 124: 2502-2506.

68. Hummell DS, Swift AJ, Tomasz A, Winkelstein JA. Activation of the alternative complement pathway by pneumococcal lipoteichoic acid. Infect Immun 1985; 47: 384-387.

69. Sato K, Quartey MK, Liebeler CL, Le CT, Giebink GS. Roles of autolysin and pneumolysin in middle ear inflammation caused by a type 3 Streptococcus pneumoniae strain in the chinchilla otitis media model. Infect Immun 1996; 64: 1140-1145.

70. Schmidt T, Täuber MG. Pharmacodynamics of antibiotics in the therapy of meningitis: infection model observations. J Antimicrob Chemother 1993; 31 Suppl D: 61-70.

71. Coonrod JD, Leach RP. Antigenemia in fulminant pneumococcemia. Ann Intern Med 1976; 84: 561-563.

72. Gillespie SH, Smith MD, Dickens A, Raynes JG, McAdam KPWJ. Detection of C-polysaccharide in serum of patients with Streptococcus pneumoniae bacteraemia. J Clin Pathol 1995; 48: 803-806.

73. Yother J, Forman C, Gray BM, Briles DE. Protection of mice from infection with Streptococcus pneumoniae by antiphosphocholine antibody. Infect Immun 1982; 36: 184-188.

74. Yother J, Volankis JE, Briles DE. Human C-reactive protein is protective against fatal Streptococcus pneumoniae infection in mice. J Immunol 1982; 128: 2374-2376.

75. McNamara MK, Ward RE, Kohler H. Monoclonal idiotype vaccine against Streptococcus pneumoniae infection. Science 1984; 226: 1325-1326.

76. Weiser JN. Phase variation in colony opacity by Streptococcus pneumoniae. Microb Drug Resist 1998; 4: 129-135.

77. Bruyn GAW, Zegers BJM, van Furth R. Mechanisms of host defense against infection with Streptococcus pneumoniae. Clin Infect Dis 1992; 14: 251-262.

78. Szalal AJ, Briles DE, Volanakis JE. Role of complement in C-reactive-protein-mediated protection of mice from Streptococcus pneumoniae. Infect Immun 1996; 64: 4850-4853.

79. Bergeron Y, Ouellet N, Deslauriers A-M, Simard M, Olivier $\mathrm{M}$, Bergeron MG. Cytokine kinetics and other host factors in response to pneumococcal pulmonary infection in mice. Infect Immun 1998; 66: 912-922.

80. Cauwels A, Wan E, Leismann M, Tuomanen E. Coexistence of CD14-dependent and independent pathways for stimulation of human monocytes by Gram-positive bacteria. Infect Immun 1997; 65: 3255-3260.

81. Riesenfeld-Orn I, Wolpe S, Garcia-Bustos JF, Hoffman MK, Tuomanen E. Production of interleukin-1 but not tumor necrosis factor by human monocytes stimulated with pneumococcal cell surface components. Infect Immun 1989; 57: 1890-1893.

82. Takashima $\mathrm{K}$, Tateda $\mathrm{K}$, Matsumoto $\mathrm{T}$, Iizawa $\mathrm{Y}$, Nakao $\mathrm{M}$, Yamaguchi K. Role of tumor necrosis factor alpha in 
pathogenesis of pneumococcal pneumonia in mice. Infect Immun 1997; 65: 257-260.

83. van der Poll T, Marchant A, Keogh CV, Goldman M, Lowry SF. Interleukin-10 impairs host defense in murine pneumococcal pneumonia. J Infect Dis 1996; 174: 994-1000.

84. van der Poll T, Keogh CV, Guirao X, Buurman WA, Kopf M, Lowry SF. Interleukin-6 gene-deficient mice show impaired defense against pneumococcal pneumonia. J Infect Dis 1997; 176: $439-444$.

85. Sande MA, Sande ER, Woolwine JD, Hackbarth CJ, Small PM. The influence of fever on the development of experimental Streptococcus pneumoniae meningitis. J Infect Dis 1997; 156: 849-850.

86. Tuomanen E, Tomasz A, Hengstler B, Zak O. The relative role of bacterial cell wall and capsule in the induction of inflammation in pneumococcal meningitis. J Infect Dis 1985; 151: $535-540$.

87. Engelhard D, Pomeranz S, Gallily R, Strauss N, Tuomanen E. Serotype-related differences in inflammatory response to Streptococcus pneumoniae in experimental meningitis. J Infect Dis 1997; 175: 979-982.

88. Täuber MG, Burroughs M, Niemoller UM, Kuster H, Borschberg U, Tuomanen E. Differences of pathophysiology in experimental meningitis caused by three strains of Streptococcus pneumoniae. J Infect Dis 1991; 163: 806-811.

89. Tuomanen E, Hengstler B, Zak O, Tomasz A. Induction of meningeal inflammation by diverse bacterial cell walls. Eur $J$ Clin Microbiol 1986; 5: 682-684.

90. Quagliarello VJ, Long WJ, Scheld WM. Morphologic alterations of the blood-brain barrier with experimental meningitis in the rat. Temporal sequences and role of encapsulation. J Clin Invest 1986; 77: 1084-1095.

91. Ernst JD, Hartiala K, Goldstein IM, Sande MA. Complement (C5)-derived chemotactic activity accounts for accumulation of polymorphonuclear leukocytes in cerebrospinal fluid of rabbits with pneumococcal meningitis. Infect Immun 1984; 46: $81-86$.

92. Täuber MG. Pathophysiology of pneumococcal meningitis. Second International Symposium of Pneumococci and Pneumococcal Diseases, Sun City, South Africa: 19-23 March 2000.

93. Sande MA, Täuber MG. Pneumococcal meningitis: current pathophysiologic concepts. In: Tomasz A (ed) Streptococcus pneumoniae: molecular biology \& mechanisms of disease. New York, Mary Ann Liebert. 2000: 315-320.

94. Ernst JD, Decazes JM, Sande MA. Experimental pneumococcal meningitis: role of leukocytes in pathogenesis. Infect Immun 1983; 41: 275-279.

95. Propp RP, Jabbari B, Barron K. Measurement of the third component of complement in cerebrospinal fluid by modified electroimmunodiffusion. J Lab Clin Med 1973; 82: 154-157.

96. Smith H, Bannister B, O'Shea MJ. Cerebrospinal-fluid immunoglobulins in meningitis. Lancet 1973; 2: 591-593.

97. Tureen JH, Täuber MG, Sande MA. Effect of hydration status on cerebral blood flow and cerebrospinal fluid lactic acidosis in rabbits with experimental meningitis. J Clin Invest 1992; 89: $947-953$.

98. Scheld WM, Dacey RG, Winn HR, Welsh JE, Jane JA, Sande MA. Cerebrospinal fluid outflow resistance in rabbits with experimental meningitis. Alterations with penicillin and methylprednisolone. J Clin Invest 1980; 66: 243-253.

99. Täuber MG. Brain edema, intracranial pressure and cerebral blood flow in bacterial meningitis. Pediatr Infect Dis J 1989; 8: $915-917$.

100. Tureen J, Liu Q, Chow L. Near-infrared spectroscopy in experimental pneumococcal meningitis in the rabbit: cerebral hemodynamics and metabolism. Pediatr Res 1996; 40: $759-763$. 